\title{
POR QUE TODO DIA É O MESMO NO ISOLAMENTO SOCIAL? QUANDO OCUPAR NÃO É PREENCHER, MAS RESSIGNIFICAR
}

\section{WHY IS EVERY DAY THE SAME IN SOCIAL ISOLATION? WHEN TO OCCUPY IS NOT TO COMPLETE, BUT REFRAME}

\author{
Daniela Sousa de Oliveira ${ }^{1}$ \\ Alan Cristian Firmo Nóbrega ${ }^{2}$ \\ George Luiz Neris Caetano ${ }^{3}$ \\ Vitória Reis Soares 4
}

1.Terapeuta Ocupacional Especialista em Saúde Coletiva, Residente em Saúde Mental do Adulto, Escola Superior de Ciências da Saúde - ESCS/FEPECS. Mestrando em saúde Coletiva pelo Universidade de Brasília. E-mail: aleinda21@gmail.com

2. Farmacêutico Residente em Saúde Mental do Adulto, Escola Superior de Ciências da Saúde - ESCS/FEPECS. E-mail: alan.nobrega93@gmail.com

3.Acadêmico de Medicina, Universidade de Brasília - FM/UnB. E-mail: georgeluiz61@yahoo.com.br

4. Acadêmica de Enfermagem, Universidade de Brasília - Faculdade de Ceilândia. Email: vitreis19@gmail.com

\section{RESUMO}

O isolamento social como medida preventiva contra a Covid-19 tem se tornado um grande desafio para as pessoas impossibilitadas de saírem de casa. As consequências deste distanciamento social já são perceptíveis, diante da suspensão de atividades ditas essenciais no cotidiano da população, que envolvem desde as ocupações desempenhadas na escola, no trabalho, na família, no lazer e no meio cultural. Com isso, ao se deparar com essa nova realidade, cabe ao indivíduo adaptar-se a este novo contexto social e utilizá-lo como oportunidade de aprendizado para ressignificar as suas relações e dinâmicas sociais, afetivas e de trabalho. Utiliza a revisão narrativa dentro de uma estrutura teórico-argumentativa. Desta forma, o presente artigo discute as mudanças 
decorrentes do isolamento e processo de fazer, organizar e realizar atividades do cotidiano. Foi possível perceber que as mudanças ocasionadas pelo isolamento social necessariamente precisam vir com a incorporação de ocupações significantes.

Palavras-chaves: Isolamento social. Terapia ocupacional. Covid-19.

\begin{abstract}
Social isolation as a preventive measure against Covid-19 has become a major challenge for people unable to leave home. The consequences of this social distance are already noticeable, given the suspension of activities considered essential in the daily life of the population, which involve since the occupations performed at school, at work, in the family, at leisure and in the cultural environment. Thus, when faced with this new reality, it is up to the individual to adapt to this new social context and use it as a learning opportunity to reframe their relationships, social, affective and work dynamics. It uses narrative review within a theoretical-argumentative framework. Thus, this article discusses the changes resulting from the isolation and process of doing, organizing and carrying out daily activities. It was possible to realize that the changes caused by social isolation necessarily must come with the incorporation of significant occupations.
\end{abstract}

Keywords: social isolation, occupational therapy, Covid-19.

\title{
INTRODUÇÃO
}

O novo coronavírus (Sars-CoV-2) foi detectado pela primeira vez na China em 2019, com o aumento de casos notificados, nesse mesmo ano, em dezembro, o país comunicou a Organização Mundial de Saúde (OMS) sobre a epidemia de doenças pulmonares, posteriormente denominada como Covid-19, causada pelo Sars-CoV-2. Em janeiro de 2020, os casos cresceram exponencialmente, ultrapassando as fronteiras asiáticas. A Europa, mais especificamente a Itália, a Espanha e o Reino Unido noticiaram o aumento de casos envolvendo doenças pulmonares, de forma muito semelhante ao que estava ocorrendo na Ásia; no mês de março, a OMS declarou pandemia internacional, por disseminação generalizada do novo vírus. O primeiro caso notificado na América Latina foi em São Paulo, no Brasil, em fevereiro de 2020. No decorrer dos meses seguintes o número de casos confirmados e óbitos causados pela Covid-19 aumentou drasticamente no país, de modo que o Brasil passou a adotar medidas protetivas e de contingenciamento, 
na tentativa de controlar a disseminação do novo vírus e diminuir a incidência da doença 12.

O isolamento social, de acordo com a OMS (2020), é um forte instrumento de proteção populacional, servindo como barreira para a propagação do novo vírus, além de ser uma política de saúde pública que visa amenizar os impactos da Covid-19 nos serviços de saúde ${ }^{3}$. Com essa medida, Brasil (2020) aponta que práticas sanitárias entram em ação em larga escala, como a atenção à etiqueta respiratória, cuidados com a higiene, inibição de aglomerações que resultam no distanciamento social ${ }^{4}$. Neste contexto, é preciso pontuar a diferença entre o isolamento social, o distanciamento social e a quarentena. $\mathrm{O}$ primeiro termo refere-se à retirada e separação do convívio social de pessoas que não compõem núcleo familiar, uma forma válida principalmente para os indivíduos assintomáticos; o segundo, é o espaçamento necessário de uma pessoa para outra, quando o isolamento não é possível, para essa medida é sugerido o distanciamento de 2 metros; e o terceiro, que é a quarentena, envolve a reclusão temporária, baseada no período incubatório do agente etiológico da pessoa infectada ou outra que manteve o contato com ela, a fim de evitar a disseminação da doença para outras pessoas ${ }^{43}$.

Dessa forma, com o cenário pandêmico da Covid-19, potencializou-se a restrição de ocupações/atividades que demandem participação coletiva. Estas são de grande relevância no tocante à funcionalidade que, segundo a Classificação Internacional de Funcionalidade e Incapacidade em Saúde (CIF), estão relacionadas com a interação entre a condição de saúde e o contexto que considera os fatores ambientais e pessoais, envolvendo uma relação dinâmica que inclui as funções e estruturas do corpo e a atividade de participação. A atual situação da Covid-19, além de gerar muitas inseguranças, tem causado interrupções abruptas nas rotinas, acarretando um custo psicossocial considerável ${ }^{6}$. 
Estudos têm apontado para a disseminação de problemáticas relacionadas à saúde mental, como quadros de depressão, ansiedade e estresse, os quais são cada vez mais frequentes na população em geral, e há relatos de casos de suicídio ocasionados pelos impactos do estado de pandemia. Tais consequências têm-se confirmado no Brasil e noutros países, uma vez que as políticas e ações públicas para conter a Covid-19 corroboram para a proteção da vida, ao passo que as medidas prolongadas e cada vez mais restritivas ameaçam a saúde mental da população ${ }^{7}$.

O isolamento social não é o único fator que tem provocado desordens na saúde mental das pessoas, visto que as questões socioeconômicas também têm afetado diretamente o bem-estar da população. Além disso, os profissionais da saúde mental estão sendo desafiados a pensar e construir estratégias de enfrentamento para a situação atual, a qual se apresenta em constante movimento e mudanças. Afirmada a preocupação da comunidade cientifica em relação às consequências da pandemia na saúde mental da população, a OMS lançou um "Guia de Cuidados para a Saúde Mental” neste período. Trata-se de um compilado de orientações para os profissionais e para a população em geral com relação às estratégias para mitigar os efeitos deletérios da pandemia da Covid19, ressaltando que as incertezas, os ricos de contaminação e a obrigação de isolamento social podem agravar e gerar problemas à saúde mental ${ }^{748}$.

Ciente dos danos que o enclausuramento pode acarretar, o Ministério da Saúde (MS) publicou uma nota técnica com recomendações à Rede de Atenção Psicossocial sobre as estratégias de organização no contexto da infecção da Covid-19. Nesta nota técnica são consideradas, para as orientações, a Lei de $n^{\circ} 13.979 / 2020$, que trata das medidas de enfrentamento à pandemia, e a Lei de $n^{\circ} 10.216 / 2001$, que reconhece os direitos das pessoas em sofrimento mental. É importante ressaltar que o estado de pandemia, além de gerar e acometer a saúde mental das pessoas funcionais, também 
acarreta um aumento no sofrimento mental daqueles que já estavam em processo de cuidados em saúde mental ${ }^{4}$.

Desta forma, este estudo se propõe a trazer reflexões tratadas na literatura atual sobre as questões provenientes do isolamento social no cotidiano, além de abordar sobre a ocupação humana como elemento de análise que norteia o fazer humano por meio de atividades, sendo essa gerada por significados e não apenas para como um preenchimento qualquer.

\section{METODOLOGIA}

Trata-se de uma pesquisa teórica e reflexiva sobre os possíveis impactos do isolamento social no cotidiano das pessoas que se mantêm confinadas ${ }^{9}$. Este artigo tem a intenção de trazer ações de problematização sobre a ocupação e o cotidiano no contexto de pandemia, contrastando com os documentos expostos na literatura atual como forma de possibilitar a construção de ideias e pequenos aprofundamentos, a fim de buscar aprimoramentos às práticas do residente multiprofissional em saúde e demais profissionais da saúde mental que compõem o programa.

Segundo Demo $(2019)^{10}$, a qualidade da metodologia está no investimento da reflexão e da prática do conhecimento, por isso, a estrutura teórico-argumentativa da construção deste artigo foi fundamentada na perspectiva da terapia ocupacional como forma de dialogar com as estruturas que fortalecem a saúde mental. Utilizou-se o método de revisão de pesquisa bibliográfica por meio da revisão narrativa, com oportunidade de trazer uma análise do estado da arte como apoio e delimitação das leituras reflexivas de artigos científicos publicados em bases de dados da Biblioteca Virtual da Saúde e do Google Acadêmico"1 12 13. Os descritores utilizados foram: “ocupação e Covid-19”, 
"isolamento social”, “ocupação e saúde", "cotidiano e pandemia", "terapia ocupacional e pandemia".

\section{RESULTADOS E DISCUSSÕES}

O trans e pós-pandemia exigirá, tanto dos profissionais da saúde mental quanto do campo social, ações e serviços individualizados e coletivos que atravessem a intersetorialidade, com a oportunidade de produção de cuidados voltados para os contextos com restrições e bloqueios das atividades/ocupações que moldam a vida e rotina das pessoas. Como forma de levantar reflexões dialógicas que respondam sobre os modelos de atenção, de ações técnicas e políticas, é importante a sintonia e responsabilidade dos trabalhadores da saúde mental, em especial, o terapeuta ocupacional (TO) que, partindo das diversas perspectivas e entendimentos do cotidiano, tem um papel essencial para a ressignificação de rupturas no cotidiano da população ${ }^{14}$.

A saúde mental tornou-se, como em outras áreas, terreno próprio de desenvolvimento de cuidados voltados para ocupações significantes, por meio das atividades de "vida diária e da participação social"15. "O terapeuta ocupacional visa melhorar as habilidades físicas, psíquicas e sociais de indivíduos frente aos desafios da vida prática"15. Vale destacar que ultimamente há um maior debate sobre as atividades e ocupações cotidianas com o fenômeno da pandemia, o que tem proporcionado grandes travessias de encontrar um tempo, espaço e ideias para o desenvolvimento das atividades $\operatorname{corriqueiras}^{15}$. A terapia ocupacional é uma categoria profissional tanto da saúde quanto do campo social, dentre outras áreas, que há muito tempo busca entender o efeito curativo das ocupações ${ }^{15}$.

Para Costa $(2013)^{16}$ o cotidiano visto pela ótica da terapia ocupacional denota subjetividade, cultura, contexto histórico e social. E é pelo cotidiano que a vida é 
estruturada, caracterizando-se numa polissemia do trabalho. As atividades e as ocupações fundamentam-se como recursos da atividade humana que impulsionam os hábitos e rotina, e isso envolve todas as atividades desempenhadas pela pessoa. Com o advento do isolamento social, as ocupações, as quais consistem no fazer, organizar e realizar, mostram-se em desordem ${ }^{16}$. A partir dessa nova perspectiva, o domicílio tem-se tornado o ambiente onde todas, ou a maioria, das ocupações são desenvolvidas. Antes, este ambiente era o lugar de outras atividades do dia-a-dia como: descanso, atividades de vida diária, lazer e socialização. Agora, este espaço está sendo, para muitos, também o local de trabalho. Esta é uma nova realidade para a maioria dos trabalhadores que têm carregado inúmeros dilemas, como a organização da rotina e o excesso de trabalho com a tentativa de ser efetivo nessa atividade, o que acarreta um grande desgaste e ansiedade aos teletrabalhadores ${ }^{16} 17$.

Esse novo cenário ocupacional se tornou uma oportunidade e a necessidade de transformar e reinventar, dentro de um ambiente e espaço, novas lógicas de trabalho, lazer, estudo, dentre outros ${ }^{16}{ }^{18}$. Os constantes desdobramentos, além do avanço da pandemia, têm mudado completamente a forma como o indivíduo está ocupando-se, de modo que essa reorganização da vida cotidiana tem causado vários problemas relacionados ao acúmulo de atividades desnecessárias pela culpabilização ou obrigatoriedade de desempenhar essas atividades para justificar o ato de "ficar em casa"19.

É certo que os impactos são consideráveis, principalmente nas atividades de integração social, de lazer e de trabalho, o que inclui ainda os projetos de vida das pessoas como: escola/faculdade, viagens, lazer, arte, cultura e religião. Sendo uma situação completamente inusitada para a maioria da população, esse contexto tem-se tornado desnaturado, de modo que a falta e sobrecarga de trabalho se torna uma dicotomia sintomática da Covid-19 16 . 
A ocupação tem uma relação com o tempo e a energia que se coloca para realizar alguma atividade, sendo necessário um planejamento baseado em um contexto. Nesse contexto, a intencionalidade é uma característica que imprime um sentido nas realizações das ocupações, pois se considera que para a execução dos papéis ocupacionais é necessário a interação entre a atividade, a pessoa e o ambiente, sob o ponto de vista das ocupações que motivem e causem propósito e significado com a perspectiva do não preenchimento $^{20}$.

A inserção de atividades como mero preenchimento tem ocasionado as pessoas que estão em isolamento social, alto grau de esgotamento, prostração e fadiga. Que podem ser evidenciados no acúmulo de atividades incompletas como também na própria sobrecarga de trabalhos ao longo do dia com ou sem organização da rotina, se revelando um grande revés para criação e desenvolvimento de habilidades adaptativas. O que gera atividades/ocupações com o único objetivo de produção, dispensando o vivenciar, a satisfação na realização das atividades. E admite com maior importância, apenas, o esforço colocado e o resulto final que podem, por sua vez, imprimir sensações de inquietudes e a exaustão subjacente à condição de estresse ${ }^{21} 22$. Segundo Biondi et al. $(2020)^{22}$, em seu estudo sobre integralidade da saúde mental em tempos de pandemia, afirma que o estresse contínuo ocasionado por uma rotina desregulada estabelece de certa forma um processo de adoecimento que tende a afetar tanto o tempo presente como também poderá ser um agravante no futuro, visto que pode estar associado às rupturas do cotidiano, como o trabalho, a escola, a participação social, dentro outras ${ }^{22}$.

Quando se está muito envolvido em preencher, pouco espaço é aberto para ocupações verdadeiramente significantes. E com o preenchimento chega-se à culpabilidade como também à ociosidade, embora entenda-se que seja fundamental refletir, organizar e executar ações com consciência e segurança, na pandemia, utilizar 
essas estratégias qualificadoras torna-se mais difícil ${ }^{18}$. Becerra-García et al. (2020) ${ }^{23}$ realizaram pesquisa na Espanha com o objetivo de investigar, por meio de questionário on-line, as questões sociodemográficas, ocupacionais e ambientais-contextuais durante a quarenta imposta pelo Estado Espanhol. Os autores chegaram à conclusão de que no período de quarentena os participantes relataram depressão, ansiedade e sensibilidade interpessoal como sintomas advindo do processo do confinamento. E que pessoas ativas e empregadas demonstram baixos sintomas depressivos se comparadas às pessoas desempregadas. A pesquisa concluiu que durante o confinamento social as pessoas estão mais vulneráveis a fatores sociodemográficos e ocupacionais-contextuais ${ }^{23}$.

Nessa situação fica evidente a importância das ocupações/atividades que tenham propósito/sentido. O contexto do isolamento social justifica-se mudanças nas atividades de vida diária, o que era habitual necessidade de remodelamentos frente a nova realidade, que é singular e que afeta cada pessoa de maneira dicotômica ${ }^{18}$. Não existe um roteiro pronto, pois esta é oportunidade ímpar de cada um desenhar para si uma rota eficiente, repleta de novos-significados, respeitando-se as limitações e acentuando as potencialidades. Independentemente de o isolamento ser colocado como uma medida temporária, a ressonância dos seus efeitos será superior ao intervalo de tempo da sua duração $^{18} 9$. As ocupações como preenchimento podem ser entendidas como um provocador sintomatológico de estresse, visto que é a forma mais comum do homem reagir ao inesperado, mas ainda em um âmbito de pandemia ${ }^{23}$. Pode acometer o prazer nas realizações das atividades e tornar o bem-estar bloqueado pela incompletude de tarefas, refletindo nas angústias e frustrações no adiamento e não cumprimento de metas advindas de um repertório empobrecido de atividades ${ }^{24}$.

É necessário pensar em resiliência em meio ao estado de pandemia, como o vivido no contexto da Covid-19, criar e executar atividades que correspondam à realidade, de 
modo que a ideia de "desocupação" possa passar de um sentimento de culpa e ou desinteresse e ser transformada em qualidade de vida. Isto contribui para que as ocupações não sejam apenas preenchidas, mas que gerem constantemente um repertório de significados para o indivíduo, baseando-se no universo que este se encontra e a volição das atividades que norteiam os desejos e as vontades da pessoa ${ }^{24}{ }^{25}$. Howarth et al. (2020) ${ }^{26}$ identificou em seu estudo que a prescrição social de soluções baseadas na natureza que promovem a saúde e o bem-estar são cada vez mais incomuns nas rotinas das pessoas que estão em isolamento social e consequentemente tem acarretado nesses indivíduos ansiedade e a obrigatoriedade de ser resiliente ${ }^{26}$.

$\mathrm{Na}$ América do Sul, mais precisamente na Argentina, Johnson et al (2020) ${ }^{27}$, em estudo que teve intenções de explorar os sentimentos e expectativas provocados pelo isolamento social, notou-se que a população relatou a incerteza, o medo e a angústia que se mistura com responsabilidade de cuidado, e que os sentimentos positivos aparecem como uma avaliação interdependente social. Afirmam ainda os pesquisadores que o vínculo entre as pessoas são atividades centrais, que merecem adaptação, tendo em vista o impacto subjetivo e social nessa conjuntura epidemiológica ${ }^{27}$. Para além de ocupações/atividades que gerem preenchimento, o estudo caracteriza que o estado motivacional do isolamento carrega inseguranças capazes de transformar tanto de forma passageira como permanente os relacionamentos interpessoais, estados emotivos e afetivos empunhado pela imprevisibilidade das circunstâncias que remotamente são visibilizadas tanto pelo descontrole das multitarefas quanto na ociosidade ${ }^{9}$. Portanto, as inúmeras atividades humanas que compõem a rotina, como: a organização do trabalho e da vida privada, o lazer e o descanso, a socialização, deve estar alinhada às necessidades e valores, crenças e afetos, pois o cotidiano é único e irrepetível para cada pessoa. 
Com isso, mesmo que o isolamento social sendo um processo imputado a todos, torna-se imprescindível o desenvolvimento de adaptações e engajamento criativo para o desempenho de atividades em um contexto diferente, para que se possa analisar mais as intenções e significados e não apenas a tarefa cumprida ${ }^{18}$. Ressaltando que a desocupação também compõe o processo do fazer humano, no sentido, de oportunizar espaços de pausas e até mesmo o momento de criação que complementam ainda mais os sentidos e significados dos processos. É necessário ater-se ao tempo em atividades como um investimento pessoal e com um propósito e significados ocupacionais ${ }^{19}{ }^{20}$. Há unanimidade quanto à realização de ocupações que estimulem as reflexões com forma de dialogar sobre estratégias que amenizem a complexidade da situação.

Sendo assim, o propósito, os motivos, os objetivos e as finalidades devem determinar as ocupações e não o preenchimento do tempo. A ocupação com significado é uma experiência subjetiva, repleta de símbolos que impulsionam o modo singular de se ocupar 201619 . Em decorrência da pandemia da Covid-19, as ocupações/atividades do cotidiano perderam ou mudaram o seu significado, devido as próprias urgências adaptativas impostas pelo momento. Nesse sentido, a ideia de ressignificar mostra-se como estratégia para a reorganização das atividades dentro de uma rotina factível e alcançáve ${ }^{18}$. Bioni et al. $(2020)^{22}$, em estudo admitiram que os grandes recursos humanos advêm de adaptações de plasticidade. Essa resposta adaptativa move as tentativas de reconstruções que podem possibilitar tanto respostas criativas e de ressignificação como também desafios. Destacam ainda que historicamente os humanos procuram se adaptar às condições difíceis para sobreviver, como o enfrentamento de destruição, ciclones, guerras, genocídios, temperaturas extremas, viver perto de vulcões ativos, terremotos que repetidamente causaram destruição ${ }^{22}$. 
Essa mudança é fundamental e, quando ressignificadas, possibilita a manutenção tanto de atividades ditas laborais, como abre espaço para a efetivação de atividades de autocuidado e lazer com maior frequência, porque os objetivos são movidos pela qualidade e não pela quantidade de ocupações ${ }^{18}$. As medidas de enfrentamento da Covid19 também estão na forma como as pessoas organizam as suas ocupações e principalmente estão relacionadas ao processo de ressignificação dessas atividades. Diante disso, a manutenção da saúde mental durante a pandemia da Covid-19 está num processo de transformação das ocupações, seja do trabalho, da escola, do lazer, da cultura, da religião e das artes. O acúmulo de atividade configura-se a partir do preenchimento delas, como forma de completar o tempo e pode representar um problema para o indivíduo, gerando ansiedade e frustração de expectativas ${ }^{189}$. Para sobreviver ao contexto imposto pela pandemia, é necessário validar as habilidades inatas como a elasticidade, a flexibilidade, a resiliência, a criatividade, a resistência, a adaptabilidade, a esperança, comportamentos em processo que busquem ocupações alinhadas à história, ao sentimento de pertença, ao planejamento, ao comprometimento, à inventividade e também à fé ${ }^{22}$.

A ressignificação não é um processo automático, mas moldado pela capacidade e desempenho na realização das atividades, envolve a subjetividade nas traduções e compreensões do fazer humano, refletindo a realidade das ocupações ${ }^{9}$. Para dar significado ao "novo normal", ao isolamento social, é preciso desenvolver mais que ferramentas, atitudes de construtividade, que se resumem em reconstruir relações de socialização, senso de comunidade, solidariedade e cooperatividade. Trata-se de atividades humanas que funcionam bem em situações de crise, trauma, uma vez que o processo de superação ou reconstrução dos significados surgem em razão do trabalho coletivo e não unicamente de um contexto individual ${ }^{22}$. 
Contudo, as ocupações significantes em sua teoria e prática consideram todas as atividades da vida humana como um processo biopsicossocial e espiritual, tomado pela capacidade e desempenho na sua realização com independência e autonomia. Desta forma, dentro dos processos terapêuticos de cuidados em saúde, trabalha-se sob a perspectiva do criar e reconstruir reflexões sobre as atividades ${ }^{18}$. No contexto de isolamento social é essencial uma visão existencial. Colocar o foco no sentido do fazer com propósito para desenvolver o sentimento de pertencimento e ser útil em algo maior ao invés de somente ater-se à quantidade de atividades concluídas. Não se pode cair na ideia de que a pandemia exige a recuperação total da rotina realizada antes de sua instalação. Deve-se reconhecer as mudanças e como também as suas respostas adaptativas, que significa investir o tempo necessário em ocupações e não em preenchimentos.

\section{CONSIDERAÇÕES FINAIS}

Para que todo dia não seja o mesmo no isolamento social é preciso ressignificar as ocupações, transformar o cotidiano e incrementar atividades de acordo com as circunstâncias. Porém, é preciso ter o entendimento de que algumas ocupações não precisam ser ressignificadas, uma vez que tal comportamento pode se tornar exaustivo. A ressignificação vem com um intuito de provocar melhoria na qualidade de vida por meio de soluções facilitadas e criativas.

É sabido que os prejuízos à saúde mental, decorrentes do isolamento social são crescentes e que a organização da rotina é importante para que não haja uma sobrecarga, quando se está trabalhando de casa. Ressalta-se que o ideal de cotidiano organizado nem sempre é aquele que gera satisfação, prazer e bem-estar, o excesso de organização também pode ser cansativo e não prazeroso. São nas ocupações que a vida acontece, e, partindo desse princípio, é fundamental o reconhecimento e pertencimento do lugar onde 
se está, para onde se deseja ir e com quem ir. A participação social intra e extrafamiliar configura melhores arranjos de suporte e desenvolvimento dos papéis ocupacionais.

A terapia ocupacional tem muito a colaborar com ações práticas e teóricas sobre o fazer por meio de atividades que possibilitam o desenvolvimento de habilidades sob o prisma da autonomia e independência. As buscas por significados devem objetivar a qualidade de vida, nesse sentido, a ocupação em tempos de pandemia deve ser instalada às rotinas maleáveis atravessadas pelo equilíbrio de atividades que mesclam o trabalho, o lazer, a espontaneidade e a criatividade, considerando sempre os contextos.

A pandemia causada pela Covid-19, para além das questões sociais, emocionais e espirituais que carrega no processo do adoecimento, amplia as questões ocupacionais com mais notoriedade por conta das consequências impostas pelas medidas que o isolamento social no cotidiano e, como um todo, em relação às atividades de socialização. Diante disso, este estudo recomenda atenção e cuidados na modelação e ressignificação das ocupações, tendo em vista os contextos e singularidades de cada pessoa. Tem em vista que a ressignificação deve ser uma oportunidade de autorreconhecimento e pertencimento como pano de fundos para propósitos e sentidos em função de viver bem. A adaptação ao contexto de pandemia torna-se essencial para desempenho saudável das ocupações. Não existe um caminho genérico em que todos possam trilhar, mas, a oportunidade de inventar e se reinventar com as ferramentas encontradas nas realidades de cada pessoa.

Nesse momento tão diferenciado é importante entender que a realidade anterior à pandemia, pode não voltar. O tão procurado "normal”, em meio à pandemia, será e é trajado com outras vestes, nas quais serão impressos cuidados tanto relacionados a autocuidado, à higiene, quanto a relações interpessoais. A grande questão que este estudo se propôs refletir não é como vamos voltar a ser como éramos, mas, como vamos encarar 
as mudanças. E como vamos dar novo sentido e significado às ocupações que já existem, ou seja, ressignificar.

\section{REFERÊNCIAS BIBLIOGRÁFICAS}

1.ORNELL, et al Pandemia de medo e CoVid-19: impacto na saúde mental e Possíveis estratégias. revista debates in psychiatry - Ahead of print 2020. Ciência \& Saúde Coletiva 2.BEZERRA, A, Silva, C.E.M, Soares, F.R.G, Silva, J.A.M. Fatores associados ao comportamento da população durante o isolamento social na pandemia de COVID-19. Cien Saúde Colet [periódico na internet] (2020/Abr). [Citado em 04/06/2020].

3.OMS. Mental health and psychosocial considerations during COVID-19 outbreak. March, 2020. Disponível em < https://www.who.int/docs/defaultsource/coronaviruse/mental-health-considerations.pdf >. Acesso em 23 de abril de 2020 4.BRASIL. Ministério da Saúde. Nota técnica nº 12/2020-CGMAD/ DAPES/ SAPS/ MS. Disponível em: < $\underline{\text { http://189.28.128.100/dab/docs/portaldab/documentos/notatecnica122020CGMADDA }}$ PESSAPSMS02abr2020COVID-19.pdf> Acesso em 24 de abril de 2020.

5.-SMITH A, Freedman DO. Isolation, quarantine, social distancing and community containment: pivotal role for old-style public health measures in the novel coronavirus (2019-nCoV) outbreak. J Travel Med. 2020 Mar 13;27(2). pii: taaa020. Doi 10.1093/jtm/taaa020.S.

6.ABRATO - Associação Brasileira dos Terapeutas Ocupacional - ABRATO. Silva DB, Oliveira PVB, Folha OAAC, Nicolau SM, Wetheimer LG, da Silva DR, Sousa RNP, Pacheco SRG, Krawczyk NOS, Moraes BM, OkumaKM, Marcolino TQ Nota da 
Associação Brasileira dos Terapeutas Ocupacionais - ABRATO sobre o COVID-19. Rev. Interinst. Bras. Ter. Ocup. Rio de Janeiro. 2020. suplemento, v.4(3): 281-289.

7.SCHMIDT, B.; Crepaldi, M. A.; Bolze, S. D. A.; Neiva-Silva, L.; Demenech, L. M. Impactos na Saúde Mental e Intervenções Psicológicas Diante da Pandemia do Novo Coronavírus (COVID-19). Estudos de Psicologia, Campinas, 2020.

8.BROOKS SK, Webster RK, Smith LE, Woodland L, Wessely S, Greenberg N, et al. The psychological impact of quarantine and how to reduce it: rapid review of the evidence. Lancet. 2020; 395:912-20.

9.GALHEIGO, Sandra Maria. Terapia ocupacional, cotidiano e a tessitura da vida: aportes teórico-conceituais para a construção de perspectivas críticas e emancipatórias. Cad. Bras. Ter. Ocup., São Carlos, v. 28, n. 1, p. 5-25, Mar. 2020.

10.DEMO, P. (2019). Metodologia do conhecimento científico. São Paulo: Atlas.

11.PRUDENTE, Francisca Geisyani Gomes et al. Atuação Fisioterapêutica no Transplante Hepático: Revisão Bibliográfica Narrativa e Integrativa. UNOPAR Cient Ciênc Biol Saúde 2015.

12.ROTHER, Edna Terezinha. Revisão sistemática $X$ revisão narrativa. Acta paul. enferm., São Paulo, v. 20, n. 2, p. v-vi, June 2007.

13.CORDEIRO, Alexander Magno et al. Revisão sistemática: uma revisão narrativa. Rev. Col. Bras. Cir., Rio de Janeiro, v. 34, n. 6, p. 428-431, Dec. 2007.

14.BREGALDA MM, Correia RL, Amado CF, Omura KM. Ações da terapia ocupacional frente ao coronavírus: reflexões sobre o que a terapia ocupacional não deve fazer em tempos de pandemia. Rev. Interinst. Bras. Ter. Ocup. Rio de Janeiro. suplemento, 2020. v.4(3): $269-271$. 
15.SILVA, DB. A Terapia Ocupacional no Brasil na perspectiva sociológica [Tese] Curitiba: Universidade Federal do Paraná, Setor de Ciências Humanas; 2017.

16.COSTA, CML et al. O valor terapêutico da ação humana e suas concepções em Terapia Ocupacional. Cad. Ter. Ocup. UFSCar, São Carlos, v. 21, n. 1, p. 195-203, 2013. 17. SILVA, DB. Terapia Ocupacional, cotidiano e pandemia COVID-19: inquietações acerca do ocupar o tempo-espaço. Rev. Interinst. Bras. Ter.Ocup. Rio de Janeiro. suplemento, 2020. suplemento, v.4(3): 529-553.

18.CARRASCO J; Olivares D. Haciendo camino al andar: construcción y comprensión de la ocupación para la investigación y práctica de la terapia ocupacional. Revista Chilena de Terapia Ocupacional. 2008.

19.CORRÊA VAC, Nascimento CAV, Omura KM. Isolamento social e ocupações. Rev. Interinst. Bras. Ter. Ocup. Rio de Janeiro. 2020. suplemento, v.4(3): 351-369.

20.LILLO SG. La ocupación y su significado como factor influyente de la identidad personal. Revista Chilena de Terapia Ocupacional. Santiago. 2003; (3): 1-6.

21.CREFITTO-8. Terapeutas Ocupacionais no enfrentamento a Covid-19 https://www.crefito8.gov.br/pr/index.php/sala-de-imprensa/noticias/2335-terapeutasocupacionais-no-enfrentamento-do-covid-19 - acessado 7 de junho de 2020.

22.BIONI, Massimo; LANNITELLI, Angela.CoViD-19 e estresse pandêmico: "Integridade mental não tem relação com estatísticas". Psiquiatria Rev 2020; 55 (3): 131136.

23.BECERRA-GARCÍA, Juan Antonio; GIMÉNEZ BALLESTA, Gala; SÁNCHEZGUTIÉRREZ, Teresa; BARBEITO RESA, Sara; CALVO, Ana. Síntomas psicopatológicos durante la cuarentena por Covid-19 en población general española: un 
análisis preliminar en función de variables sociodemográficas y ambientalesocupacionales. Rev Esp Salud Publica; 942020 Jun 09.

24.MIRANDA EFS, Santos LBS, Santos JM, Oliveira PVB. Cotidianidades frente ao Coronavírus em uma residência em saúde: possibilidades construídas junto a terapeutas ocupacionais. Rev. Interinst. Bras. Ter. Ocup. Rio de Janeiro. 2020. suplemento, v.4(3): 488-495.

25.TEDESCO SA. Avaliação e intervenção de terapia ocupacional em contextos hospitalares. In: DE CARLO, M. M. R. D. P.; KUDO, A. M. (org) Terapia Ocupacional em contextos hospitalares e cuidados paliativos. Editora Payá, 2018; p. 80-102

26.HOWARTH, Michelle; GRIFFITHS, Alistair; SILVA, Anna; GREEN,Richard.

Social prescribing: a 'natural' community-based solution. British Journal of Community Nursing June 2020 Vol 25, No 6.

27.JOHNSON, María Cecilia; SALETTI-CUESTA, Lorena and TUMAS, Natalia. Emociones, preocupaciones y reflexiones frente a la pandemia del COVID-19 en Argentina. Ciênc. saúde coletiva [online]. 2020. 